This is an Accepted Manuscript of an article published by Taylor \& Francis in Cataloging \& Classification Quarterly on November 6, 2019, available online:

https://doi.org/10.1080/01639374.2019.1680470

Version of Record: Kristi Bergland, Kalan Knudson Davis \& Stacie Traill (2019) CatDoc HackDoc: Tools and Processes for Managing Documentation Lifecycle, Workflows, and Accessibility, Cataloging \& Classification Quarterly, 57:7-8, 463-495, DOI: 10.1080/01639374.2019.1680470

\title{
CatDoc HackDoc: Tools and Processes for Managing Documentation Lifecycle, Workflows, and Accessibility
}

\section{Kristi Bergland, Kalan Knudson Davis, \& Stacie Traill}

\begin{abstract}
Documentation of local policies, workflows, and procedures is an important activity for cataloging and metadata units. But creating and maintaining documentation is a huge task that is not always a high priority. Librarians at the University of Minnesota Libraries planned a documentation hackathon, CatDoc HackDoc, with three primary goals: to update a large amount of documentation quickly, to apply accessibility best practices to all documentation, and to bring new staff into the documentation workflow. This article describes the event's planning process, structure, and outcomes, and offers guidance on how others can adapt the CatDoc HackDoc model in their own organizations.
\end{abstract}

Keywords: cataloging, metadata, documentation, accessibility, workflows, hackathons

\section{Introduction}


Documentation of local policies, workflows, and procedures is an important activity for library cataloging and metadata units, no matter how large or small. Accurate, up-to-date documentation facilitates staff learning, serves as a record of institutional memory, encourages consistency, and simplifies and improves the accuracy of analysis projects based on catalog data. However, creating and maintaining documentation is a huge task that is often not a high priority in day-to-day work, especially when staff already feel overloaded, and it may be difficult to justify the need for documentation work to administrators who are focused on production.

When attention does turn to creating documentation, it is often viewed as a onetime project, rather than as a process of continuous review, revision, and refinement. Documentation, especially in the maintenance portion of its lifecycle, is usually not a high priority -- that is, until just before a key staff member departs or retires. The writing of cataloging documentation is often dependent on an individual or small group of managers or subject matter experts having time to write documentation. When they do occur, major documentation efforts are often tied to a system migration or standard implementation -- situations in which there are likely to be numerous other high-priority tasks competing for staff resources. What, then, is the rationale for devoting substantial staff resources toward documentation? How can we make a strong case for documentation as an ongoing focus?

Probably the most compelling reason to prioritize documentation is to ensure that it is an accurate and reliable tool for library staff to both learn new concepts and tasks and review familiar ones. Staff turnover is inevitable, and even long-term staff are likely to see their job responsibilities change over time. Good documentation can ease such 
transitions, both by increasing staff confidence and improving process efficiency. When good documentation is in place, new staff members should not have to "reverse engineer" procedural steps or invent (and document) an entirely new procedure where a previously undocumented one had been in place.

Another critical function of documentation is to encourage consistency of practice. This is especially true when more than one staff member is responsible for the same or similar cataloging and metadata tasks. Staff who began working for the library at different times may have learned their jobs under different standards and practices or may have learned their jobs under different supervisors or lead workers. As job responsibilities, metadata standards, and library systems all change over time, it is challenging to keep everyone "on the same page." Ongoing attention paid to documentation can help alleviate this problem. ${ }^{1}$

Accurate, up-to-date documentation is also a valuable tool for data analysis. Five decades after the invention of MARC, and 30-40 years after many libraries implemented their first integrated library system, the need for documentation showing how library data practices have changed over time is acute and growing. Thoughtfully maintained documentation can explain puzzling elements in legacy data, help identify areas for data remediation, and serve as an informal registry or key for analysis projects drawing on library metadata.

Whatever the rationale for prioritizing documentation, it is important to think of documentation as an ongoing process, rather than as a project with a clearly defined endpoint. Good documentation has a lifecycle. The components of the documentation lifecycle may vary slightly, but a simple lifecycle might include drafting, publishing, 
reviewing, and updating. Conceptualizing documentation in this way means that the commitment of resources to it must be ongoing, rather than time-bounded (although the amount of time needed for documentation work varies throughout the lifecycle).

As staff in the Data Management and Access department (DMA) of the University of Minnesota Libraries (UL) with substantial assigned responsibility for cataloging and metadata documentation, we were committed to creating and maintaining good documentation, but we struggled to find the time and resources to manage documentation effectively. Beginning in 2016, we undertook a department-wide initiative to review and revise existing documentation and identify needs for new documentation. We used this as an opportunity to rethink our entire documentation workflow, resulting in the creation of a recurring annual event: CatDoc HackDoc, which informs and feeds ongoing documentation management processes. This article will discuss the development of the CatDoc HackDoc idea, along with its influences and goals. It will also discuss how we took advantage of this opportunity to align documentation work with UL- and campus-wide initiatives to improve accessibility. We will also describe the planning process for the annual CatDoc HackDoc events, the structure of the events themselves, their outcomes, and the role they play in the documentation lifecycle. Finally, we will provide some guidance on how others can adapt the CatDoc HackDoc model for their own organizations. The University of Minnesota Libraries places strong emphasis on collegial collaboration, sharing resources, responding to needs in innovative ways, creating partnerships, and developing new models and services. CatDoc HackDoc and the tools and processes enabled by it align closely with these broader organizational goals. 


\section{Background}

The University of Minnesota Libraries serve the flagship Twin Cities campus of the University of Minnesota system. UL, comprising 12 libraries, shares a common cloud-based library services platform (Alma) and discovery layer (Primo) with the separately administered Law Library and libraries located on the other campuses in the University of Minnesota system. The Twin Cities campus population includes approximately 20,500 FTE undergraduates, 14,500 FTE graduate and professional students, and 18,000 faculty and staff. In 2012 and 2013, UL undertook three major initiatives in a short span of time, which had the unintended consequence of severely disrupting documentation activities in technical services.

In 2012, UL underwent an internal reorganization, resulting in the splitting of the Technical Services department into two separate departments in different divisions. The Data Management and Access department (DMA) united cataloging and metadata staff with the former library systems department, while the Acquisitions and E-Resources Management department (ARM) incorporated its namesake functions. The reorganization disrupted the model of technical services documentation management that had previously been in place, resulting in a lack of clarity about both central coordination of documentation efforts, and which staff were responsible for documentation.

At the same time, the University of Minnesota system adopted Google Suite as an enterprise solution and began moving away from the previous campus-wide network storage utility, Netfiles. UL technical services documentation formerly stored in Netfiles 
was uploaded to Google Drive as static PDF or Microsoft Word files, typically without revision or change in file format.

Finally, in 2013, UL implemented Resource Description Access (RDA) for cataloging, and also completed a library system migration from Ex Libris's Aleph to the Alma library services platform. Staff created or revised many documents focused on copy cataloging as part of RDA implementation, but did not have adequate time to revise all existing documentation to comply with the new cataloging guidelines. At the same time, staff created and revised a large amount of policy and procedure documentation related to library system functionality as part of the Alma migration project. Because local RDA implementation and Alma migration had different timelines, staff involvement, and project scopes, a comprehensive effort to revise all existing documentation was not possible, although DMA department leadership recognized it as a need.

On the heels of the initiatives described above, in mid-2014, UL offered a Voluntary Layoff Option, which resulted in the departure of four staff members with cataloging responsibilities. Because of these departures, some cataloging and metadata documentation described processes and workflows that were no longer maintainable at current staff levels. Some documentation even referenced staff members by name who were no longer employed by UL.

By 2015, it had become clear that we needed a substantial, sustained effort to return cataloging and metadata documentation to a baseline where all documentation had been reviewed for content accuracy, converted to a common file format, stored in a single known location, and could be easily found and used by staff. 


\section{Inventory and assessment}

With fewer staff members to do the work and new online tools available to support a more collaborative documentation model, the UL Cataloging and Metadata Group (CMG) began to think broadly about how local cataloging and metadata documentation could be more effectively presented, accessed, and organized. However, the goal was to do more than a one-time review, reformatting, and reorganization: we wanted to create a sustainable model for assessment, content review, and ongoing documentation work in order to stay close to the documentation baseline going forward, once it had been re-established. As with many documentation projects, we began by creating an inventory and assessing current documentation. What was less clear was how to proceed once the assessment phase was complete. Revision of all the documentation identified during the assessment phase seemed like an overwhelming task.

Seeking a collaborative and sustainable way forward, CMG decided to pursue a team-driven solution that could solve the immediate problem of completing revisions in a timely manner while being more responsive to near-term documentation needs and sharing documentation work among more staff. The CatDoc HackDoc was a way to utilize Agile/Scrum-inspired practices in the context of a documentation "hackathon" to make documentation everyone's priority for an intensive two-day event.

\section{Documentation accessibility}

Although our initial motivation was to devise a new process and tools for documentation creation and maintenance, we were also concerned with documentation 
accessibility -- something that had never previously been an explicit concern in UL's cataloging documentation work. Having effective, usable documentation is an accessibility issue as well as an equity, diversity, and inclusion issue. At its simplest, accessible documentation is a resource that brings everyone to the same starting point. It need not be seen as an accommodation, even though discussion of accessibility is often framed around disability. At the core of accessibility in documentation is a focus on the learning outcome and styling each document to support that goal. When the design and presentation of a document enhances rather than distracts, the user is able to focus on the content.

The Association for Library Collections and Technical Services (ALCTS) names "recruiting and retaining people who bring a range of perspectives to technical services" ${ }^{2}$ as a practice vital to the future of the profession. Providing colleagues with the tools they need to do their jobs seems like a minimum requirement, but viewed from the perspective of a new employee, it can be a strong predictor of how welcome they will feel in a new environment. Recording and communicating local practice can be a way of making newcomers feel part of their new community as well as serving as a training tool.

Hodges points out that within technical services, there are "innumerable circumstances where diversity and inclusion are determined by ordinary professionals going about their everyday work." ${ }^{3}$ Creating and revising documentation is part of our everyday work. Including many people in the documentation revision process not only ensures that a variety of perspectives are represented, but also gives many individuals the chance to apply accessibility principles hands-on. The benefits of this collaborative 
process are immediate. Everyone has current, useful documentation, and all benefit as both users and creators of the work. Emphasizing accessibility was therefore a perfect fit for our broader goals as we developed the CatDoc HackDoc model.

\section{Literature review}

In a 2006 article, White defines documentation as anything written down in a department that pertains to the present, past, or future operation of the library and can assist in clarifying and confirming the nature of library activities. ${ }^{4}$ White notes that although documentation is incredibly important to technical services, finding information within the professional library literature on the topic can be frustrating. The literature does contain some case studies describing lessons learned from documentation projects and best practices for documentation creation. Projects generally start with an analysis of the current state of documentation, followed by an organization process and well-intentioned plans for ongoing review. Where the case studies leave off is describing how to achieve an ongoing, holistic documentation review cycle. Broadly interpreted, the literature on technical services documentation suggests adopting the following general practices, which informed our choices:

- Make documentation readily available and easy to find

- Proactively consider documentation accessibility

- Use plain language

- Use image and design purposefully

- Gather feedback from users of documentation

- Use appropriate technology and tools

- Incorporate documentation practice into daily work 
- Share the load and involve everyone in the process of creating and maintaining documentation.

White indicates that the health of a technical services environment and the library organization itself can be assessed by the state of its documentation. Documentation encourages communication and staff working openly together as all use their individual skill sets to improve the library. ${ }^{5}$

\section{Issues identified in case studies}

Falk, Hertenstein, and Hunker note that staff retirements, the implementation of a new discovery layer, advances in technology, and staff reductions drove their need for collaboration among existing cataloging staff to assess and formulate documentation for local cataloging practices. ${ }^{6}$ Staff reductions led to a loss of departmental history and created a void in communication and documentation practices. The staff changes also required all remaining cataloging staff to be aware of upcoming changes in existing procedures. A lack of up-to-date procedures caused difficulty with new staff learning local practices. White also notes that processes and procedures often rely on institutional memory or a partial understanding of larger library processes, and states that very few staff members actually find time to include creating documentation within the "department's daily repertoire of activity." Schmitt and Barstow suggest that a lack of documentation can lead to employee problems and even lawsuits, result in slower processing for library materials deemed objectionable, and cause problems with work assignments. ${ }^{8}$ Scheschy observes that "written procedures are an essential tool for training new staff," emphasizing that "[o]ral tradition" is unreliable for making local decisions, and that every member of the technical services department should be 
encouraged to participate in writing down and describing their daily activities in completing tasks. ${ }^{9}$

\section{Assessment}

Plummer and Rigda discuss the preliminary assessment and considerations that need to take place before technical services documentation is created. ${ }^{10}$ These include time commitment, participation, project support, hardware and software issues, and training needed related to using the new manual. ${ }^{11}$ White recommends making time every day for documentation. ${ }^{12}$ This work is seen as a reflective activity and an opportunity to improve processes. White sees documentation as an essential part of a healthy technical services environment and recommends this be seen as "everyday" work rather than a "frantic, burdensome extra." 13

\section{Tools and organization}

White notes that very early on in the adoption of online tools, technical services departments began to place policy and manuals online. ${ }^{14}$ The professional literature describing early online adoption focuses mainly on tools and usage of those tools rather than best practices. Falk, Hertenstein, and Hunker discuss the creation of an online and comprehensive cataloging manual by examining existing documentation technical services and special collections documentation. ${ }^{15}$ This cataloging manual was to accomplish two goals: training a new cataloger and documenting current policies and procedures. First, an assessment of the available documentation was completed. Legacy workflows and processes were also assessed while creating new workflows. Where incomplete instructions were identified, step-by-step instructions with screen 
captures supplemented explanations. Meetings with catalogers and discussions ensured a variety of departmental input. Catalogers self-selected in reviewing and wikirelated tasks. Next, the revision tasks were assigned to those with cataloging and format expertise. A wiki was created to store working documents, and the documentation was compiled together into one document to give a consistent look and feel. Finally, the authors made decisions on where the manual would reside and maintenance required by an ongoing review cycle. ${ }^{16}$

Norton describes using Google-based tools to document policies and procedures for the University of Michigan Library Michigan Publishing department. The process began with an inventory and assessment of existing resources, decisions on which online systems to use, the creation of an organizational framework for the site, and other knowledge gathering activities. The team identified ongoing steps to maintain, update, and add additional content going forward using a distributed staff-driven model; the development of best practices and standards for the maintenance of the content; and usage of the Intranet version control functionality. ${ }^{17}$

Plummer and Rigda describe a process they used to develop a comprehensive cataloging procedure manual that would be dynamically updated and interactive. ${ }^{18}$ The process of creating this procedure manual included preliminary considerations, such as time commitment, departmental support, and hardware/software issues. They participated in a research phase to understand the literature surrounding transitioning print procedures manuals to an online format; gather information on departmental needs; and examine existing cataloging documentation sites. The next steps included site design, organization, increasing familiarity with W3C Web Content Accessibility 
Guidelines, and user testing. Surveying the cataloging staff using a short questionnaire gave further insight into the upkeep, design and ongoing maintenance of their cataloging documentation resources.

Black and Stalberg describe using staff shadowing to document cataloging workflows due to staffing changes in their metadata and cataloging department. The workflow analysis initiative included compiling field notes regarding the serials lifecycle. Their job shadowing project "increase[d] knowledge and empathy across staff members and encouraged career exploration." 19 The shadowing sessions provided an opportunity for staff members to observe a breadth and depth of cataloging work and see the workflows in real time. The project helped raise awareness of processes and reduced inconsistency, inefficiency and confusion for staff members. Regular meetings addressing the department's workflow challenges helped achieve a smoother and more efficient processing of materials.

Faulkner remarks on the complexity of library workflows and suggests that charting a workflow visual can reveal inefficiencies and unnecessary handoffs between staff members. Specifically, Faulkner discusses the Lucidchart browser-based application, which eases flowchart creation. The intuitive interface and logical placement of menus eases user understanding of the Lucidchart software. ${ }^{20}$

Evans, Intner, and Weihs recommend written policy decisions on cataloging and classification. The authors suggest that written policy ensures continuity in staffing changes. Manuals should be linked to larger library missions and goals, arranged in a logical fashion, and address specific library material formats. It is not only important to 
record the policy decision made, but also the reasons that support the policy decision. Ongoing review of policies informs policy changes that may be needed. ${ }^{21}$

\section{Accessibility and usability}

Accessibility in technical services documentation is not well addressed in the existing literature. Turner and Schomberg address improving the effectiveness and usability of library documentation in general by applying the principles of Universal Design for Learning (UDL), Gestalt theory, and the Plain Language movement. ${ }^{22}$ One UDL principle is to consider the learning objective for the document, and how design choices help meet that objective. They illustrate both problematic and improved examples of the Gestalt principles of figure-ground segregation, closure, proximity, continuity, similarity, past experience, and symmetry/equilibrium as they relate to library documentation. It is also important to use plain, direct language with minimal use of jargon, and to provide enough context that the document user does not have to spend excessive time and mental energy just to get to the starting point of the document. They also stress the importance of usability testing to ensure that the documentation truly speaks to its intended audience.

Beyond that, the literature on documentation accessibility falls into two main categories. The first comprises studies about the needs of specific user groups or style resources to address those needs, while the second comprises style guides and resources for designing accessible web content. Rello and Marcos present the results of an eye tracking study comparing reading performance and user preferences in customization of text settings and conclude that beyond improved contrast and larger 
text size, there is no clear recommendation for other variables in improving reading comprehension. ${ }^{23}$ Rello and Baeza-Yates provide recommendations of larger fonts, high contrast, and larger, strategic page spacing to increase reading speed of people with dyslexia. ${ }^{24}$ In their study of the reading speed of people with dyslexia of various common fonts, Rello and Marcos also found that font choice has an effect on reading speed and performance..$^{25}$ Sans serif, roman, and monospaced fonts increased reading performance, and Helvetica, Arial, Courier, and Verdana are particularly recommended. The Dyslexia Style Guide 2018 of the British Dyslexia Association recommends Arial and Comic Sans, followed by Verdana, Tahoma, Century Gothic, Trebuchet, Calibri, and Open Sans in a font size of $12-14 .{ }^{26}$ They also recommend increased spacing, avoiding italics and underlining, use of "headings and styles to create consistent structure to help people navigate through content", left-aligned text, use of active voice, and direct, concise sentences. In presenting design considerations for visual learning resources for neurodivergent students, Brown recommends avoiding visual glare through lower intensity colors, ensuring proper contrast, establishing visual hierarchy, using icons, and using legible fonts such as Helvetica, Verdana, Arial, Comic Sans, Garamond, or Consolas. ${ }^{27}$

The other category of literature comprises style guides and resources for designing accessible web content. Although there are differences in design needs of web content, many of the principles used are equally useful in document design. Web Content Accessibility Guidelines (WCAG) have become the recognized standard for making web content more accessible to people with a wide range of disabilities. The guidelines are structured around four principles: the resources must be perceivable, 
operable, understandable, and robust. ${ }^{28} \mathrm{~A}$ comprehensive checklist maps the success criteria under each principle.

The United States government publishes the Plain Language Handbook to support the implementation of the Plain Writing Act of $2010 .{ }^{29}$ Originally created for the U.S. Securities and Exchange Commission, the Handbook contains a style guide for plain writing to produce concise, well-organized writing created with its audience in mind. A valuable part of this resource, useful to writers in any discipline, is the extensive selection of examples of all types of writing, including samples of before and after rewrites.

\section{Agile/Scrum, sprints, and hackathons}

The Agile methodology and Scrum process framework are very popular project management methods for software development. They have generated substantial interest in other areas, including libraries. Agile is an "umbrella term for a set of frameworks and practices based on the values and principles expressed in the Manifesto for Agile Software Development and the 12 Principles behind it." ${ }^{30}$ Agile emphasizes collaborative, self-organizing teams, user focus, and iterative development as key elements of software development processes. Agile is meant to increase adaptiveness and enable teams to respond effectively to fast-paced change. Scrum is one of several process frameworks aligned with Agile values and principles. In Scrum, product development work is split into multiple two- to four-week iterations, known as "sprints." ${ }^{11}$ Each sprint picks up where the last left off and incorporates both planning and continuous review. 
Practitioners outside the realm of software development have frequently attempted to adapt Agile and its associated frameworks, including Scrum, for use in their projects. Library technical services departments are among the organization types that have explored and implemented elements of Agile and Scrum. Collins and Wilson discuss an adaptation of the Agile framework in use in Technical Services at North Carolina State University (NCSU) Libraries. ${ }^{32}$ Collins and Wilson describe how NCSU has adapted Agile for operational management in technical services, including iterative planning and decision making, clear and lightweight project management tools, and workflow mapping and analysis. They identify several positive outcomes: improved communication across all levels of staff, ability to deploy resources more readily, greater staff involvement in process management, and ability to quickly address changing priorities. ${ }^{33}$ Dean, Landaverde, Lorenzo, and Smeltekop described how Michigan State University (MSU) Libraries have incorporated Agile/Scrum into their work, creating cross-departmental teams collaborating on software and metadata projects. ${ }^{34}$

Like Agile/Scrum, hackathons originated in the domain of software development. A hackathon, defined broadly, is "creative problem solving" in the context of "any event of any duration where people come together to solve problems." ${ }^{35}$ Unlike Agile/Scrum, hackathons may last anywhere from several hours to a few weeks, and involve any number of participants who self-organize into temporary teams to work on previously defined problems or projects. Libraries have successfully adopted the hackathon model to address domain-specific problems. One especially well-documented approach is the Islandora Collaboration Group's (ICG) "Hack/Doc" model. ${ }^{36}$ According to ICG's Hack/Doc GitHub repository, "A Hack/Doc differs from a regular hackfest in its focus on 
a variety of skills beyond programming, including documentation, research, and testing." 37 ICG's model served as a primary inspiration for UL's CatDoc HackDoc cataloging documentation events.

\section{Before CatDoc HackDoc: inventory, assessment, planning}

By early spring 2018, UL's Cataloging and Metadata Group had completed an inventory and assessment of UL's local cataloging and metadata documentation and faced the problem of how to proceed. The inventory and assessment process produced a spreadsheet of existing documentation, including links to the online locations of each document and information on document "owners," the staff members who held primary responsibility for content. During the assessment process, CMG members reviewed each document, made notes on what kinds of revisions were needed, and assigned the document to one of five categories indicating the extent of revision required:

- Minor revisions needed

- Major revisions needed

- No revisions needed

- Obsolete (no longer needed)

- Revisions complete.

The assessment gave us a much-needed clear picture of the current state of our documentation, but it did not naturally suggest a way forward. The inventory and assessment process itself had taken more than eighteen months. We knew we needed to find a much faster way to accomplish the revisions and reorganization of documentation. 
The idea for CatDoc HackDoc had two sources: a weeklong experimental web development sprint held at UL in 2017, in which several DMA department staff participated, and the "Hack/Doc" events held by the Islandora Collaboration Group (described above). UL's development sprint was organized and run as a weeklong Agile/Scrum sprint, and DMA participants had generally positive feedback. As we considered how to meld these inspirations into our own event and process, we recast some of Agile's 12 Principles to suit the needs of documentation work. ${ }^{38}$ We attempted to incorporate these principles into both our hackathon-sprint as well as our ongoing documentation workflow:

- Individuals and interactions over processes and tools

- Functional and accessible documentation over perfect documentation

- Team collaboration over top-down approaches

- Responding to change over following a plan.

Additionally, the ICG's GitHub repository, with extensive documentation and templates for event planning, was an extremely valuable resource for us as we planned the first CatDoc HackDoc event.

\section{Workflow and logistics planning}

The planning for the initial CatDoc HackDoc event took place over a three-month period beginning in March 2018. The initial meetings examined the ICG's Hack/Doc model and brainstormed how it could be adapted for our work environment and needs. Pre-event planning included logistics and event planning tasks, such as reserving rooms and other work spaces for the event, food, drinks, reserving laptops, power 
cords, extension cords, ensuring network access, ordering office supplies (post it notes, sharpies, whiteboard markers), making hotel arrangements for out of town guests (catalogers from other University of Minnesota campuses), and creating a communication plan. Before the event, we organized the cataloging documentation reviewed during the prior assessment process into pre-populated work folders in Google Drive. We decided to focus work during the event on three categories from the initial assessment: Minor revisions needed, No revisions needed, and Revisions complete.

Documents identified as needing major revision were set aside for post-event work. The daily schedule for the event was adapted from ICG's Hack/Doc planning documents. ${ }^{39}$ The initial CatDoc HackDoc event took place in June 2018.

\section{Accessibility planning}

In preparation for the event, we made accessibility choices supported by the literature on accessible documentation, with the understanding that documentation is a process rather than a fixed state, and that some situational flexibility would be needed. Keeping this in mind, we created a set of formatting guidelines for participants to refer to during the event. The literature supports increased font size and generous spacing between sections to establish hierarchy, so we decided to use Calibri size 14 font across all documents for normal text with spaces buffering each section. We evaluated graphics in all documents and removed the ones that were not necessary, especially screenshots that might become quickly outdated. The rest were updated to reflect the current library system and every image was described in an alternate text (alt-text) interpretation. Sometimes the best solution was to convert graphics entirely into text, 
and many tables were built fresh in the new document to solve residual formatting issues. These changes resulted in a more uniform appearance to our documentation, but also extended the overall length of many documents.

Other changes included scanning the documents for use of direct, concise language, eliminating jargon, expanding abbreviations at first use, using headers and paragraph styles for consistency and ease of use with screen readers, adding tables of contents for longer documents, and removing distracting headers, footers, and pagination.

\section{The CatDoc HackDoc teams and process}

The initial CatDoc HackDoc comprised four functions, each handled by one or more teams: Content (two teams), Accessibility, Organization, and Sites. The goal of the Content teams was to revise documents designated during assessment as needing "Minor revisions." The Accessibility team's goal was to proofread, clarify language, and apply consistent and accessible formatting to the documents revised by the Content teams. The Accessibility team's work folder also contained copies of documents that CMG had determined needed no revisions in the documentation assessment process. The Organization team's goal was to move the revised and formatted content from the working copies provided by the Content and Accessibility Teams to their official destinations in DMA's shared Google Drive space, in which CMG had already created a folder structure to organize documentation according to cataloger needs. 
Path:_LIB-TC Staff Drive > L LIB-TC Divisions and Departments $>$ Data \& Technology $>$ Data Management \& Access

Metadata

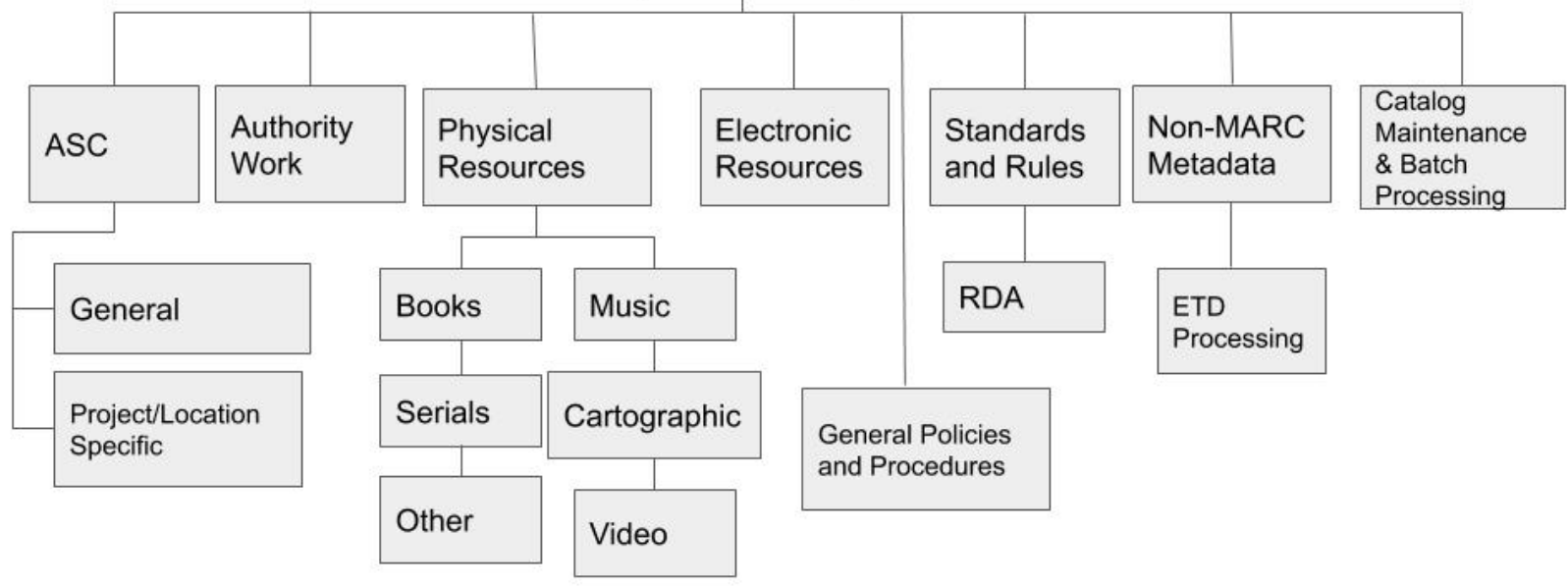

Figure 1. Cataloging documentation folder structure in Google Drive.

The Organization team also created a spreadsheet of URLs for the Sites team to populate the library staff website. This workflow ensured that the URLs used as previous website links, personal bookmarks, and links between documentation would not change. Finally, the Sites team's goal was to create a new cataloging and metadata staff webpage by populating it with links to the revised documentation provided by the Organization team. At the same time, the Sites team was superseding outdated Word and PDF versions of the documents using Google Drive's built-in versioning tools and adding URL redirects to the updated documentation. 


\section{CatDoc HackDoc : a cataloging documentation hackathon.}

Thursday, June 14 and Friday, June 15, 2018

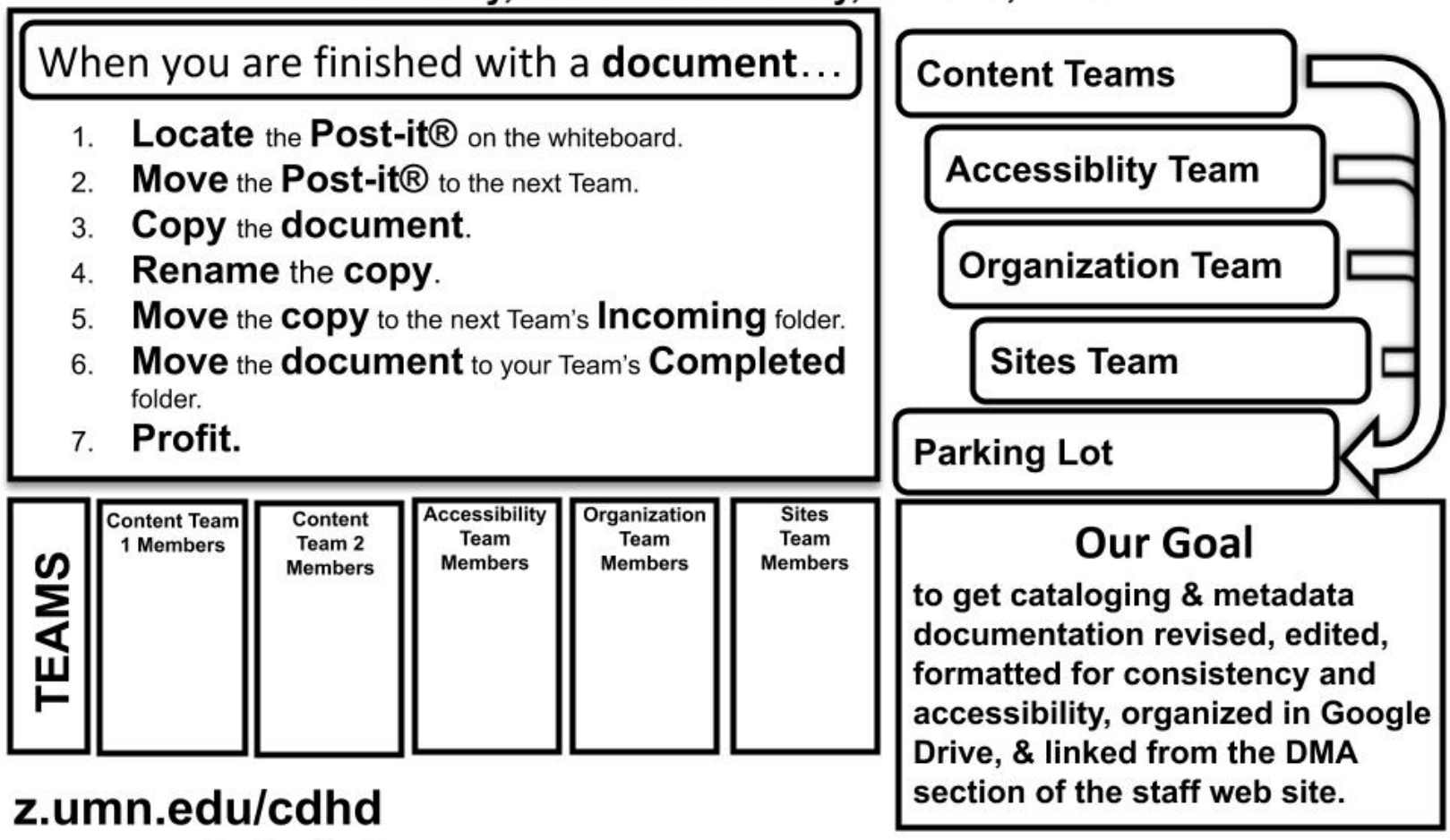

\section{z.umn.edu/cdhd-process}

Figure 2. Teams and process for the first CatDoc HackDoc. A version of this diagram was posted during the event for participants' reference.

Throughout this process, if a Content or Accessibility team member decided that a document required extensive, time-consuming work, or had questions that could not be quickly resolved, they would place that document in a "Parking Lot" Google Drive folder, ensuring that the overall process could keep moving. The "Parking Lot" served as a waypoint for documents requiring more complex revisions to be revisited after the two-day event.

To ensure that no content was lost during the event, the Content and Accessibility teams worked from "Incoming" folders pre-populated with copies of 
cataloging documents. Each team in turn populated the next Team's work folders by copying, renaming, and moving files into the Incoming folder of the next team. This workflow allowed teams to seamlessly hand off documents.

We decided to use a physical kanban board to track progress during the course of the event. A kanban board visualizes work as a series of states or processes that flow sequentially toward the team's goal. ${ }^{40}$ Our simple kanban board contained columns for each team and the parking lot, with each document title on a sticky note to enable moving them from column to column.

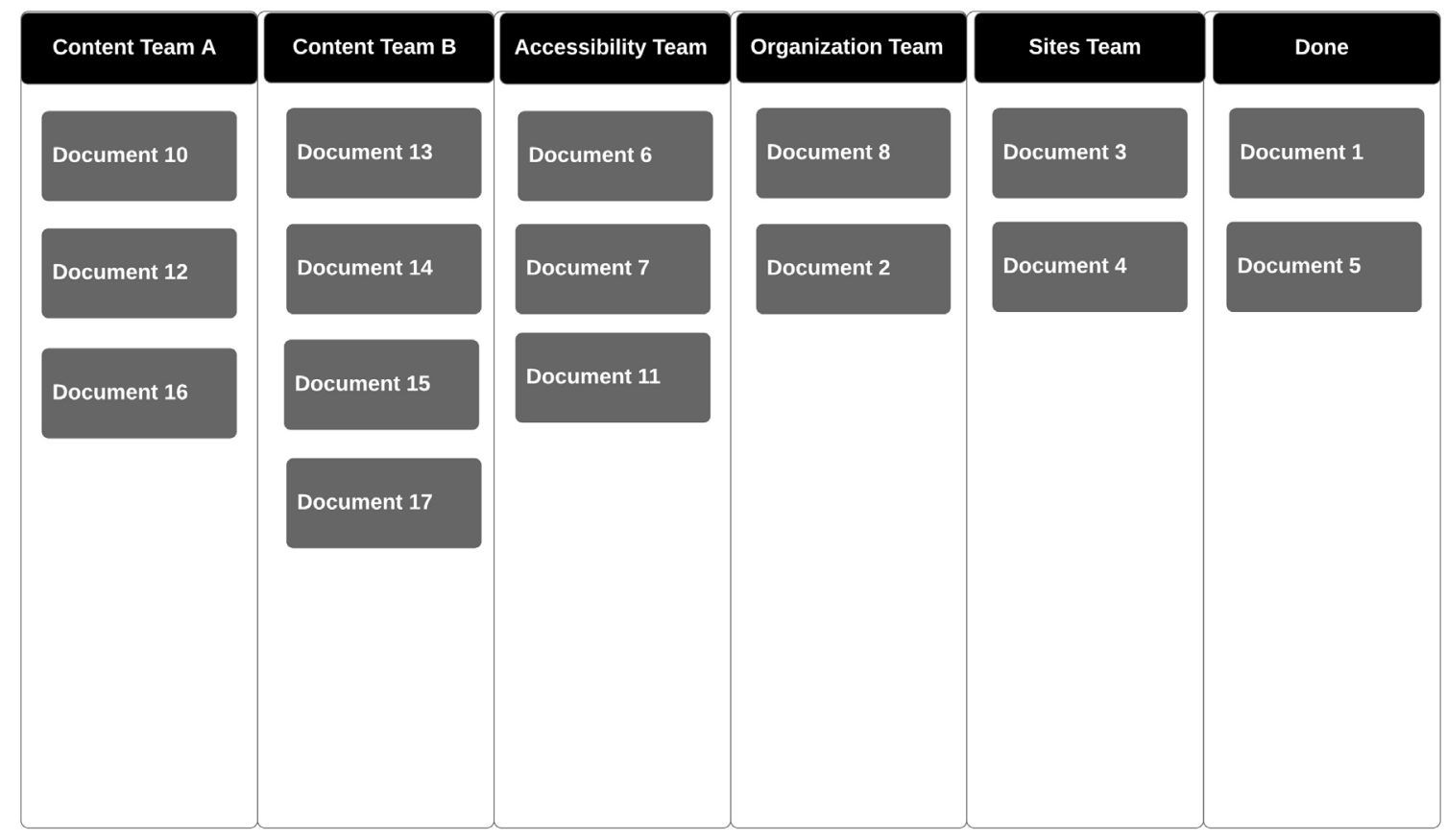

Figure 3. CatDoc HackDoc kanban board layout. The kanban board was drawn on a whiteboard and individual documents were represented on sticky notes. 


\section{Day of the event}

On the day of the event, we arrived early to finalize the setup of the room, laptops, and kanban board. Participants included most DMA department members, along with three catalogers from University of Minnesota system campuses located outside the Twin Cities. We also invited interested observers from other UL departments. After an event kickoff by the department director, the event conveners introduced the CatDoc HackDoc process, described the roles of the various teams, and explained the process from start to finish. The leaders of each team introduced themselves, and participants self-sorted into the four teams. The vast amount of documentation revisions required splitting up documentation between two different Content teams, each playing to the team leader's expertise and strengths.

The bulk of the daily agenda for each day of the CatDoc HackDoc was devoted to several breakout sessions during which teams could work on their assigned documents in whatever order they chose. At the end of each morning and afternoon, all participants convened together, and teams reported out on their progress. The daily agenda also included opportunities for individuals to discuss how the process was working and how the process could be improved. Planned breaks kept participants fresh on tasks, well fed, and watered.

The kanban board was a successful tool, keeping team members motivated and allowing everyone to track overall progress at a glance. As teams finished editing, formatting, organizing, and linking a document, its sticky note was moved from one column of the kanban board to the next, often resulting in small impromptu celebrations in the room, especially when a document moved to the "Done" column. The kanban 
board also helped us understand where work was backlogged, so we could ask participants to shift from one team to another. For example, the Accessibility team found itself with a dauntingly large number of documents in its column at the end of Day 1. The kanban board made the Accessibility backlog obvious, and participants shifted tasks to work through it. As the two days of the event progressed, participants' sense of accomplishment grew as more and more sticky notes moved through the various teams to the "Done" column.

At the end of the event, each team gave a final report on their accomplishments. Participants reflected on lessons learned, what worked well, and what could have gone better. We also collectively brainstormed a list of 33 new ideas for documentation needed and began to prioritize those ideas as a group.

\section{Post-event assessment}

After the conclusion of the 2018 CatDoc HackDoc, we created and shared a Google Form to invite feedback from event participants. Overall, event participants enjoyed the energy and enthusiasm generated from working on usually tedious documentation tasks as a group. Participants particularly mentioned the ability to track progress throughout the event using the kanban board. They also appreciated how event workflows made document handoffs between teams work smoothly. Inclusion of non-Twin Cities campus catalogers built collegial relationships and raised morale. For some staff, it was the first opportunity they had had to work 'in person' rather than remotely with staff from other campuses. 
We also solicited ideas on how the event could be improved next time.

Suggestions from participants included having better instructions on using Google Docs, and having clear checklists and guidelines for both Content and Accessibility teams. We were able to incorporate these suggestions into the 2019 CatDoc HackDoc event.

\section{CatDoc HackDoc outcomes}

At the end of the first day, participants had completed 17 documents, moving them through revision, reformatting to meet accessibility guidelines, organization in Google Drive, and linking on the new webpage. At that point, most of the Content teams' work was complete. As a result, many of these members self-reassigned to the Accessibility team, demonstrating one way in which the CatDoc HackDoc could be agile and responsive to bottlenecks in the workflow.

By the end of day two, another 77 cataloging documents in the CatDoc HackDoc had been completely updated. Over the course of the event, 11 documents were placed in the Parking Lot. A few documents went into the Parking Lot, but were able to have their issues resolved, and moved through the remainder of the process. Event attendance was very high, with 21 of the 26 invited staff participating at some point over the two days. As a bonus, 15 documents tagged as needing "major revision" that were not originally part of the event workflow were examined by Content Team leaders to determine next steps.

The CatDoc HackDoc enabled us to undertake a holistic and comprehensive assessment of our documentation, to prioritize the future documentation work-cycle, and to introduce staff to best practices for documentation accessibility. Staff members 
gained a sense of ownership over the documentation and a better understanding of the scope of work that the department collectively manages. Most importantly, we achieved our primary goal: to return to a cataloging documentation baseline with a healthy and up-to-date corpus of cataloging documentation. The ways in which we progressed toward these goals are detailed below.

\section{Better organization and linking}

During the CatDoc HackDoc event, we added 38 redirects from outdated *.doc and *.pdf files using Google Drive's version history feature. Because superseded Word and PDF versions of documents might have been bookmarked by staff and linked from various other places, we decided they could not be deleted outright. Using Google Drive's built-in versioning abilities, we replaced these documents with a message and a link redirecting users to current versions of documentation. The redirects send users to the most current version of documentation available without breaking any pre-existing links. Since the initial 2018 CatDoc HackDoc, we have added hundreds of redirects, rerouting documentation from outdated versions, duplicate copies, and obsolete documentation.

\section{Improved documentation accessibility}

For the first CatDoc HackDoc, one formidable hurdle was to synthesize the widely varying styles of four separate eras of departmental documentation, each containing its own formatting quirks, many of which frustrated Accessibility team members encountering them. In some cases, it proved easier to build a new document 
from scratch than to clear out all previous hidden formatting from an existing one. Despite the challenges, by the end of the event, all of the documents except those routed to the Parking Lot had been updated to meet our basic accessibility guidelines, including the addition of meaningful alternate text (alt-text) for images. Adding alt-text not only made images machine readable, but also served as a reminder to review the choice of image, ensuring that the image actually communicated the intended message.

Some of our practices evolved during the course of the event. Sometimes the best practices for certain user groups were at odds with each other, or principles applied to one screen of the document at a time conflicted with the overall legibility of the document. As we worked, we found it preferable to have multiple options for font style and size to improve the overall appearance, rather than to adhere strictly to a style sheet while ignoring the visual presentation and overall usability of a document. For example, font size for text within tables could be adjusted to allow a table to fit on a single page rather than breaking the table across multiple pages. We continued to refine our accessibility guidelines after the 2018 CatDoc based on staff feedback as they used documentation in the course of their daily work.

\section{Team and skill building}

The CatDoc HackDoc event was also a team and skill building exercise. The division of labor into various functional teams allowed staff members to work together in self-selected and self-directed units toward a common goal. Participants could collaborate with others with whom they may not routinely have the opportunity to work. Teams also relied on one another's expertise with Google Suite for help with editing, 
formatting, and organizing. Many participants were able to enhance their skill and comfort levels with Google Docs, Google Drive, and Google Sites in ways that they had not been able to via classroom learning and individual experimentation. The event also increased awareness among participants of cataloging and metadata workflows they might not normally utilize in their day-to-day work, resulting in a greater overall understanding of the many and varied tasks undertaken by DMA department members in the course of their everyday work.

\section{External resources and sharing}

In addition to organizing UL's local cataloging and metadata documentation in one place, we included links to external cataloging resources, tools, and other staff training resources on the centralized cataloging documentation webpage. Event participants suggested a number of tools and resources for the webpage that CMG had not previously considered.

We also adopted the practice of setting Google Doc permissions to "Anyone on the Internet can find and view" (except for those documents containing restricted or sensitive data) with the goal of increasing the findability and visibility of our documentation both inside and outside UL. This practice supports UL's active participation in collaborative and consortial cataloging and collection management initiatives, making it easy for us to share our documentation with consortial partners and other collaborators. 


\section{Including non-catalogers}

The flexibility of the CatDoc HackDoc process enabled staff who are not primarily catalogers to participate due to the many avenues of contribution. Although it may seem counterintuitive, non-cataloging staff could contribute even to the Content teams without experience with the procedures and policies documented. Non-departmental staff and non-catalogers could participate in the event by following the content and accessibility guidelines, style sheets, best practices, and checklists. In many cases, non-cataloger staff offered extremely valuable suggestions, because they viewed documentation from a non-specialist perspective and therefore could more easily identify confusing jargon and unnecessary complexity. Finally, CatDoc HackDoc also helped the non-cataloging staff who participated gain a greater appreciation for the value and complexity of cataloging and metadata work.

\section{Agency and ownership}

One goal of the initial 2018 CatDoc HackDoc event was to develop a sense of ownership over documentation among all cataloging and metadata staff, not just those who had previously written and maintained it. We want staff to feel that they have agency in how their everyday work processes are documented: they can and should suggest changes and additions, point out when writing is unclear, and even draft new documentation for processes and procedures not already covered in existing documentation.

Participants in the initial 2018 CatDoc HackDoc offered generally positive feedback about the event and felt good about their contributions to it. However, some 
staff expressed hesitation to suggest changes to documentation. Some of this hesitancy can be attributed to the novelty of writing and revising documentation, for the many staff who had not regularly done this work in the past. In addition, some staff did not feel they were expert enough to contribute to documentation (even for processes they had been working with for several years). We continue to emphasize that documentation is a living thing, subject to a continuous process of revision, and that everyone can help make documentation better and more usable for others. However, this conception of documentation -- as an ongoing process in which everyone's contributions are valuable and necessary -- represents a significant culture change, and it will take time for the change to permeate the organization.

\section{Guidelines, tools, and workflows for ongoing documentation work}

Another outcome of the 2018 CatDoc HackDoc event was the development of a set of guidelines and recommended practices for the department's documentation. These guidelines, along with accessibility templates and a style sheet, ensure that we can develop documentation as consistently and accessibly as Google Docs will allow. The guidelines outline how to manage the documentation lifecycle. They include:

- How and where to create new documents

- How to update and revise existing documents

- How to shepherd a document through the appropriate governance groups for library-wide (or system-wide) approval

- How to manage documents in the cataloging documentation Google Drive space, including setting ownership and sharing permissions 
- How to update links in documentation and on the staff webpage

- How to handle drafts, outdated versions, and obsolete documentation

- How to alert staff to new and revised documentation when it is available.

These guidelines and templates provided the foundation for our ongoing documentation workflow, which we refined, clarified, and restructured after the 2018 CatDoc HackDoc.

\section{Supporting development of cataloging competencies}

The American Library Association maintains a wide variety of knowledge and competencies statements developed by various professional organizations. ${ }^{41}$ The Core Competencies for Cataloging and Metadata Professional Librarians supplements the broader Core Competencies of Librarianship. ${ }^{42}$ The Core Competencies for Cataloging and Metadata Professional Librarians cover two broad areas: skill and ability competencies, and knowledge competencies. One of the skill and ability competencies is "application of universal standards within a local context," which is a central goal of local cataloging and metadata documentation. By reinforcing for staff how national and international cataloging and metadata standards are applied in a local context and including them in the process of documenting local decisions, the CatDoc HackDoc supported the development of this competency. Another example of "application of universal standards within a local context" in the Core Competencies is "designs and modifies cataloging and metadata workflow processes." The CatDoc HackDoc supported this competency by encouraging staff to think critically about whether existing 
documentation adequately reflected our workflow processes and to suggest changes where needed.

Cataloging and metadata staff who participated in CatDoc HackDoc also had the opportunity to develop knowledge competencies. Carefully and thoughtfully reviewing documentation increased participants' understanding of conceptual models, cataloging tools, and encoding standards. They also gained knowledge of how UL's library systems function with regard to metadata and discovery.

\section{CatDoc HackDoc: responsive adaptation}

The success of the first CatDoc HackDoc left no question that we should hold another event. The second CatDoc HackDoc event took place in June 2019. Because we had achieved our main goal of returning to a healthy documentation baseline in the 2018 CatDoc HackDoc, the identified needs, scope and deliverables of the 2019 event were different from those of the 2019 event. Instead, the major goal of the second CatDoc HackDoc was to create a sustainable and collaborative model for content review, while strengthening our commitment to accessible documentation.

During the 2018 CatDoc HackDoc, we had brainstormed a list of new documentation needs. Part of the planning for the second CatDoc HackDoc was to prioritize and clarify those needs. The 2019 event debuted a new team named "Skeledocs" (skeleton documents). The Skeledocs team's goals included informal outlining of portions of participants' regular work that did not currently have documentation and creating "skeleton" documentation for areas where crucial and pressing documentation needs had been identified. Focusing on the creation of an 
informal high-level outline that could be expanded and developed later, Skeledocs was a successful attempt to make the process of creating new documentation less overwhelming.

We changed our approach to documentation accessibility in several ways during the planning of the 2019 CatDoc HackDoc. For example, while our original font and font size choices (Calibri 14) improved the readability of individual portions of the text, the larger font size and extra spacing meant that less content was viewable on a single page. It was actually more difficult to determine the intended hierarchy of the content at a glance.

Simply by adjusting font style and size to Arial 12 for normal text and being more deliberate about spacing, the first document revised went from 16 to 11 pages. Even though it was a document our group had been working on for several weeks, when the formatting revisions were shared for review, the feedback returned was about the content, not the formatting. When the formatting issues were addressed, the focus was on the substance of the document. Streamlining the organization of information revealed subtleties of the content that even a group of subject experts had missed.

We observed that many of the tables of contents, generated from section headings at all levels, were very long and too granular, sometimes exceeding the first full page of a document. We pruned out the unnecessary detail, keeping only the top few levels. In the previous CatDoc HackDoc we removed pagination, but in the 2019 event we added it back in, responding to user feedback that it was too easy to get lost in a lengthy document without page numbers. Another change in approach was to allow 
for some flexibility in font, font size, and spacing within tables, as we prioritized avoiding splitting tables across pages over rigid uniformity.

The University of Minnesota has a resource called Accessible U, sponsored through the University of Minnesota Disability Resource Center, which "seeks to empower University students, staff, faculty, and guests with information and strategies for creating a more inclusive, accessible $U$ of $M$ community." ${ }^{43}$ This resource seeks to help all levels of the University community incorporate accessible design elements into their works, whatever format and style that may take. By demonstrating the principles behind six core skills: headings and document structure, hyperlinks, video captions, bullets and numbered lists, color and contrast, and image alt-text, Accessible $U$ supports skill-building in effective digital communication for all users. This resource was very useful to us in revising our style sheet and accessibility guidelines for the second CatDoc HackDoc event, as it stresses the importance of flexibility and that there is no single solution for all users.

The following list highlights the main actions taken for reviewing our documentation in the Accessibility review in the second CatDoc HackDoc event. This list is derived from our Accessibility checklist (Appendix A) and style sheet (Appendix B). Some of the checklist elements we applied to all documentation include:

- Convert landscape to portrait orientation

- Review document for clarity of language, spelling errors, typos

- Remove headers and footers

- Use headings and paragraph styles

- Left align all text 
- Convert normal text style to Arial, font size 12, Calibri as alternate choice

- Use bullet-pointed lists rather than prose

- Scan document for MARC subfield delimiters, convert variants to " $\$$ "

- Review spacing - single spaced preferred, remove extra space before or after paragraph as needed to establish visual hierarchy

- Add meaningful alt text to graphics and images

- Scan document for URLs and convert URLs to descriptive text hyperlinks

- Add hyperlinked table of contents to longer documents

- Check URLs/hyperlinks in document, verify that they still work

- Add pagination

- Add version history statement at bottom of document.

One important way in which we incorporated the feedback gathered after the 2018 CatDoc HackDoc was by creating new and lower-barrier avenues for participation, promoting the use of checklists and workflows for team members, and streamlining hand-offs between teams. In addition to the Accessibility checklist (Appendix A), we also created a simple Content checklist (Appendix C) to guide staff in what to look for as they reviewed content. During the CatDoc HackDoc 2, we surpassed our original figures from the initial 2018 event. In preparation for the event, we identified 99 documents for the CatDoc HackDoc 2 workflow. We completed 90 documents and aligned them with our updated accessibility standards. 35 of these 90 documents also received a full content review. As in the previous year's event, staff used the 'parking lot' to flag documents that needed extensive additional work. At the end of the event, eight 
documents were in the parking lot. One document was identified as obsolete. Finally, participants created eight "skeledocs," each providing a foundation for full documentation of previously undocumented processes.

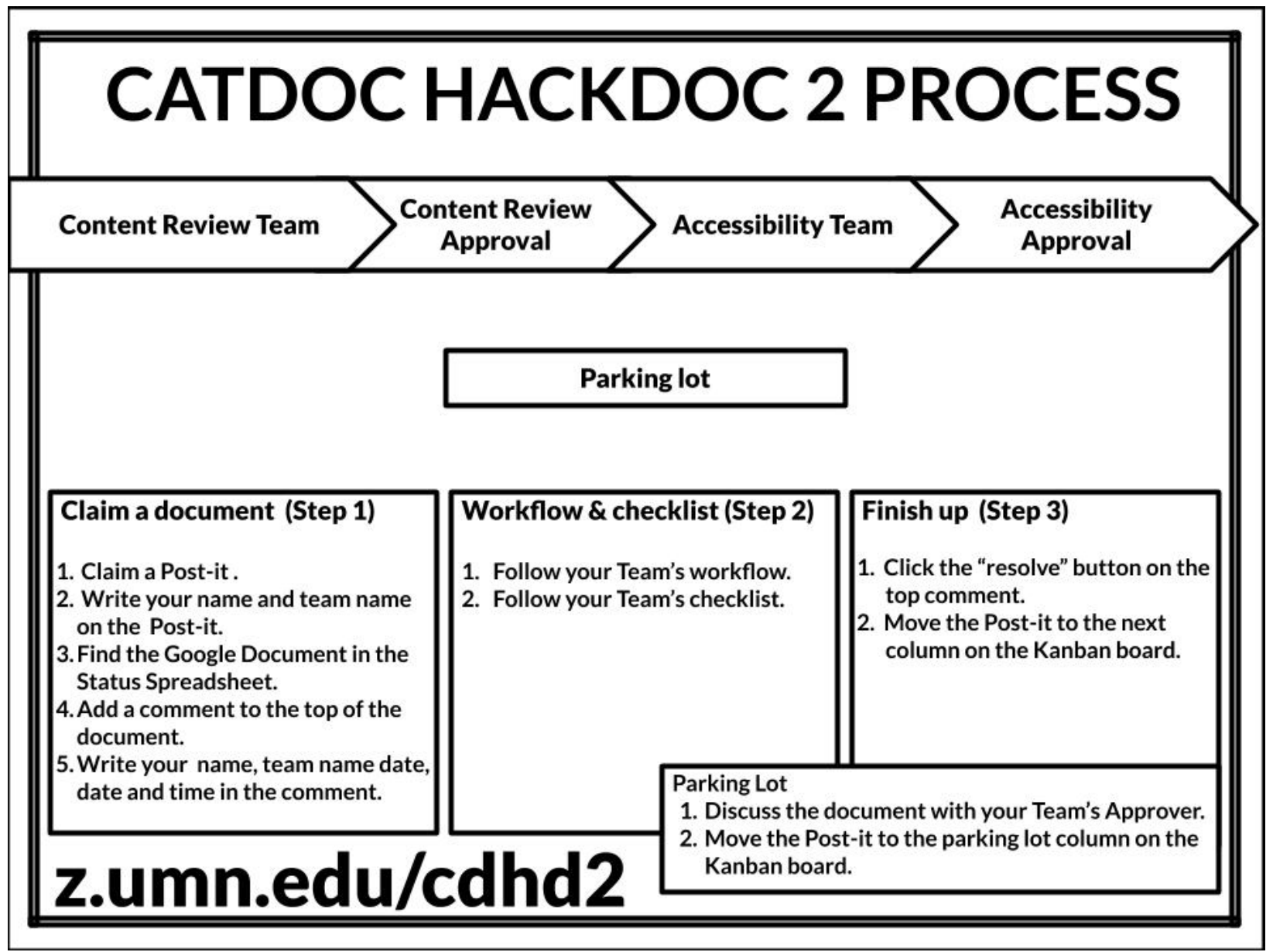

Figure 4. Teams and process for the second CatDoc HackDoc. A version of this diagram was posted during the event for participants' reference.

Once again, we asked participants to submit feedback about the event via a Google Form. Participants were again very positive about the event. In particular, they indicated that they appreciated having checklists for content and accessibility, and the additional avenues for participation. 
The success of the 2019 event demonstrated that CatDoc HackDoc could be a sustainable annual event going forward. As in the previous year, we will use the 2019 event's outcomes to inform the documentation work of CMG for the coming year, including both resolving questions raised in parking lot documentation, and enriching the skeledocs created during the 2019 event. With a second annual CatDoc HackDoc successfully completed, we are well on our way to achieving a department-wide understanding of documentation as an ongoing process to which everyone contributes.

As a postscript to the second successful CatDoc HackDoc event, we have been asked to present our work and advise other UL departments on planning their own documentation hackathon events: an "AcqDoc HackDoc" for acquisitions documentation and a "CircDoc HackDoc" for circulation documentation.

\section{Building a model for the documentation lifecycle}

Following the 2018 CatDoc HackDoc, we re-established CMG's documentation subgroup, the members of which are all subject matter experts who have documentation as a core responsibility of their positions. The documentation subgroup meets at least quarterly and maintains a Trello board (virtual kanban) to track the progress of documents through drafting, review, accessibility and style formatting, and approval by relevant UL committees. In late summer 2018, the documentation subgroup devised a plan, prioritization, and timeline to handle the documents flagged in the original assessment process as needing major revision, as well as those placed in the Parking Lot during the CatDoc HackDoc. Most of those documents were successfully revised and their issues resolved before the 2019 CatDoc HackDoc. We rely on our 
Trello board as both a tracking system and communication tool, using it to assign tasks, manage due dates, and keep CMG aware of the documentation subgroup's current work priorities.

Although our various responsibilities and organizational structure do not allow the documentation subgroup to work as a dedicated Agile/Scrum team, we are incorporating some Agile/Scrum ideas and practices. For example, frequent check-ins help maintain individual accountability and keep us realistic about timelines. The concept of iterative enhancement helps us view documentation writing as a development process and mitigates the tendency to want documentation in a perfect, finished state before it is shared. Understanding that documentation has a lifecycle encourages us to attend to it regularly and proactively, as opposed to occasionally and reactively.

\section{Sharing the CatDoc HackDoc model}

One of our goals in sharing so much detail about the planning, process, and outcomes for CatDoc HackDoc is to offer a model that can be adapted to other organizational contexts, especially for institutions smaller than ours. Our endeavor was ambitious, with a large number of people moving a large number of documents through several processes in a short amount of time. However, the basic principles behind preparation, organization, and workflow can be adapted for use in any size department or organization.

Before starting to plan a documentation hackathon, think about and share your guiding principles and high-level decisions for documentation. These decisions may 
include the level of detail expected in local documentation, when it is preferable to rely on external documentation (e.g., Library of Congress/Program for Cooperative Cataloging documentation or system vendor documentation), and what accessibility policies and practices documentation should meet.

It may also be necessary to complete an inventory and assessment project before planning a documentation hackathon, to clarify both the scope of work and the specific tasks you wish to accomplish during the hackathon itself. Whether an inventory and assessment are part of the preparation or not, clarity of the scope of work for the event is essential. The scope should be specific about what documentation will be handled during the event, and what should be dealt with at other times. Revision or writing tasks that are likely to be very time-consuming should be handled outside the documentation hackathon in order to maximize how much can be accomplished during the hackathon itself. In-scope documents should be assigned to meaningful categories before the event, which are linked to specific tasks or activities to be completed during the event.

During the event, checklists are an indispensable tool for several reasons: they encourage consistency across documents handled by different people, they help participants feel comfortable working on documentation when they have limited experience doing so, and they clarify and reinforce overall priorities (i.e., accessibility). If possible, offer ways for participants to increase their comfort levels with your organization's technological tools for documentation management before the event, and offer non-judgmental assistance as needed during the event. Offer multiple modes of participation with the goal of making all participants feel like they can contribute 
meaningfully. Let participants self-select into groups or teams rather than assigning them.

Finally, think about how to assess the success of your event, and seek feedback from participants. Consider how the event will feed ongoing documentation work and how that work will be accomplished between events.

\section{Conclusion}

In early 2018 , we faced the seemingly insurmountable task of updating, organizing, and maintaining a large local corpus of cataloging documentation. Among our many challenges were an internal library reorganization, the redeployment of a smaller cataloging staff, an enterprise-wide adoption of Google Suite, the adoption of a new library services platform, and the implementation of RDA, all of which had left our documentation disorganized, scattered across multiple storage systems and document formats -- frequently with outdated or obsolete content. We needed to find an efficient way to get back to a documentation baseline.

After completing an inventory and assessment of the state of local cataloging and metadata documentation, we decided to try a hackathon model patterned after the work of the Islandora Collaboration Group, and the CatDoc HackDoc was born. CatDoc HackDoc had several ambitious goals: to update many documents in a short amount of time, to apply accessibility best practices to all of our documentation, and to bring staff into the process of creating and maintaining documentation who were not accustomed to that type of work. CatDoc HackDoc achieved all of these goals, and also helped us 
determine a new way to structure the ongoing documentation work cycle between annual CatDoc HackDoc events.

The library literature emphasizes repeatedly that documentation is better when it is created collaboratively, with extensive staff engagement. Documentation work is never done, but this work can and should be shared by all. With CatDoc HackDoc, our hope is that we have created a sustainable and adaptable model to facilitate better, more accessible, more up-to-date documentation created with greater staff engagement. We now understand CatDoc HackDoc as more than just a one-time event: it is an overarching philosophy and approach to managing documentation over its entire lifecycle, as a process rather than a project. 


\section{Appendix A: Accessibility Checklist}

\section{Accessibility Checklist}

Remove old style format from revised documents:

- Remove headers/footers

- Remove Regents copyright statement

- Remove "Magnifying Glass (Example)" icon and text

- Remove bookmarks (not the same as hyperlinks!)

- Update to portrait orientation, if landscape

Update documents using the DMA Procedures Style guidelines:

Body

- Add pagination (number should be in upper right corner, numbering begins on first page-may need to remove old pagination first, if revising)

- Insert->Header \& Page number-> Page number

- Use Headings in:

- correct size

- correct font

- Indent outline/heading structure in Table of Contents, etc.

- Indentations: left flush, no hanging indents

- Use bulleted lists (rounded bullets preferred)

- Spell out acronyms and abbreviations the first time they appear in a document

- Use concise, meaningful text for hyperlinks within document

Fonts

- Use sans serif fonts (refer to the style sheet for examples)

- Use (minimum) font size 12 for regular text

- Use font size 12 for Table of Contents

Spacing

- No spaces after headings

- Line spacing is 1.15

- Minimize spacing before and after paragraphs

- Use a single space between sentences

- No large gaps (white space) between sections of documentation content (e.g. new section beginning on next page)

Images and Examples

- Minimize the use of images, especially screen shots of Alma (with the understanding that sometimes they are necessary)

- Use meaningful alt-text for all images

- MARC subfield delimiters used are \$\$

- MARC field examples should be in Courier New, font size 12

- Use bulleted lists (rounded bullets preferred) 


\section{Language}

- Scan for consistent use of plain language, as outlined in style guidelines. Move document to Parking Lot if extensive changes are needed.

Version History:

- Add or revise Version History statement as necessary:

- Version History statement appears roughly 1 inch/3 returns after the text of the document

- Has Version history reviewed statement: Reviewed and revised by DMA staff June 13, 2019

- Version history issued date: month (spelled out), day and year 
Appendix B: Style Sheet

\section{DMA Procedures Style Sheet}

This resource outlines the branding and style choices that will guide any documentation work for the Data Management and Access department.

\section{Fonts}

Text should be large enough to be read by most people, but small enough that information can be presented concisely. Use headings in paragraph styles rather than manually adding them.

Sans serif fonts preferred.

Minimum font size: Arial 12 or, alternately, Calibri 12 for regular text

\section{Title (Title) - Calibri Bold, size 24}

Headings (Heading 1) - Calibri Bold, size 18

Subheadings (Heading 2) - Calibri Bold, size 16

Subheadings (Heading 3) - Calibri Bold, size 14

Subheadings (Heading 4) - Calibri Bold, size 12

Narrative text - Arial, size 12 or Calibri, size 12

Version history - Arial size 12

\section{MARC tags}

Use Courier or Courier New, size 12, (bold optional)

Our practice is to use $\$ \$$ rather than $\$$ for subfield delimiters

For shorter bits in body of text, Arial size 12/Calibri size 12 (i.e. matching the existing text) may be used.

\section{Indentation}

Use left flush indent, no hanging indents

Outline/heading structure (in Table of Contents, etc) should be indented

\section{Orientation}

Portrait orientation is preferred to landscape 


\section{Spacing}

Use 1.15 spacing between lines

Use 1 space between sentences

Minimize use of spacing before and after paragraphs, enough to establish visual hierarchy, but no large gaps (white space) between sections (e.g. new section beginning on next page)

\section{Color Palette}

Maroon (R122, G0, B25) or (\#7a0019)

Blue/Gray (R223, G223, B223) or (\#e1dfdf)

\section{Table of Contents}

Where it makes sense to use a Table of Contents, keep it concise. Only include the essential sections.

Use font size 12 .

\section{Pagination}

Use pagination. Number should be in the upper right corner, numbering begins on first page (Insert->Header \& Page number-> Page number)

\section{Plain Language}

Use plain language: short sentences in active voice with Subject-verb-object construction.

Avoid complicated sentence construction.

Scan for personal bias and remove it where possible.

Remove nonessential information.

Documentation should include clear, concise information. Ask yourself:

- What is missing?

- What is unneeded?

- Does this make sense to someone not already familiar with the information? Spell out acronyms and abbreviations the first time they appear in a document. Use bulleted lists (rounded bullets preferred)

\section{Hyperlinks}

Use concise, meaningful text for hyperlinks within documentation, rather than "click here" or simply pasting the linked URL. 


\section{Images and Alt-text}

Avoid using Alma screen shots. They might seem useful in the short term, but as Alma is updated frequently, this means documentation may be quickly outdated.

Use meaningful alt-text for all images.

\section{Use common sense}

This style sheet presents options. Choices that lead to a readable, usable document are preferred to a strict interpretation of "the rules". Choosing a different font may allow information to fit in a text box better or allow an entire table to display on one page.

When in doubt, ask!

\section{Version History}

At end of document, leave one inch gap from end of text

For documents with existing version history information:

Version History:

Issued: February 11, 2010

Revised: September 5, 2016 by Mary Huismann

For anything that doesn't have existing version history worked on in CatDoc HackDoc:

Version History:

Reviewed and revised by DMA staff June 14, 2019 


\section{Appendix C: Content Review Checklist}

\section{Content Review Checklist}

\begin{tabular}{|c|c|c|}
\hline$\nabla$ & Check for: & Action to take: \\
\hline & References to AACR2 & $\begin{array}{l}\text { Make Suggestion: RDA toolkit } \\
\text { check }\end{array}$ \\
\hline & References to LCRIs & $\begin{array}{l}\text { Make Suggestion: RDA toolkit } \\
\text { check }\end{array}$ \\
\hline & References to Aleph in text & $\begin{array}{l}\text { Make Suggestion: replace with } \\
\text { Alma (or more extensive rewriting, } \\
\text { depending on context) }\end{array}$ \\
\hline & Aleph screenshots & $\begin{array}{l}\text { Make Suggestion: Aleph screenshot } \\
\text { to be deleted or replaced }\end{array}$ \\
\hline & Screenshots of old Alma UI & $\begin{array}{l}\text { Make Suggestion: old Alma UI } \\
\text { screenshot to be replaced or } \\
\text { deleted }\end{array}$ \\
\hline & $\begin{array}{l}\text { URLs/links within the document to } \\
\text { make sure they work }\end{array}$ & $\begin{array}{l}\text { Make Suggestion: find the correct } \\
\text { URL if possible, and suggest it. If } \\
\text { correct URL can't be found, suggest } \\
\text { removing the URL. }\end{array}$ \\
\hline & $\begin{array}{l}\text { Terminal punctuation in MARC field } \\
\text { examples for } 2 X X, 3 X X, 4 X X, 5 X X, 740\end{array}$ & $\begin{array}{l}\text { Delete terminal punctuation in } \\
\text { MARC field examples for tag } \\
\text { groups mentioned }\end{array}$ \\
\hline & $\begin{array}{l}\text { Check for obsolete MARC tags that } \\
\text { aren't clearly indicated as obsolete } \\
(400,440,590)\end{array}$ & $\begin{array}{l}\text { Make Suggestion: Review and } \\
\text { possibly replace or delete }\end{array}$ \\
\hline & $\begin{array}{l}\text { References to individual staff } \\
\text { members (names or initials), especially } \\
\text { if they are no longer here }\end{array}$ & $\begin{array}{l}\text { Make Suggestion: Review and } \\
\text { possibly delete }\end{array}$ \\
\hline
\end{tabular}




\begin{tabular}{|l|l|l|}
\hline Any language that's unclear to you & $\begin{array}{l}\text { Make Suggestion: Review for } \\
\text { clarity }\end{array}$ \\
\hline $\begin{array}{l}\text { If you're familiar with the } \\
\text { procedure/policy: anything that } \\
\text { doesn't match your understanding of } \\
\text { how we do things/what our policy is }\end{array}$ & $\begin{array}{l}\text { Make Suggestion: what you think } \\
\text { needs to be revised/clarified }\end{array}$ \\
\hline
\end{tabular}

\footnotetext{
${ }^{1}$ Karen A. Plummer and Christine Rigda, "Creating an Online Cataloging Manual: a Case Study," Technical Services Quarterly 22, no.2 (2004): 29, https://doi.org/10.1300/J124v22n02_03.

${ }_{2}^{2}$ Association for Library Collections and Technical Services, "About Us," accessed June 6, 2019, http://www.ala.org/alcts/about\#diversity.

3 Dracine Hodges, "Pursuing Diversity and Inclusion in Technical Services," Serials Review 44, no. 3 (2018): 172, https://doi.org/10.1080/00987913.2018.1549888.

${ }^{4}$ Hollie C. White, Documentation in Technical Services, The Serials Librarian, 49, no.3 (2006): 47-55. https://doi.org/10.1300/J123v49n03_04.

${ }^{5}$ White, 54.

${ }^{6}$ Patricia K. Falk, Elizabeth Hertenstein, and Stefanie Dennis Hunker, "Catalogers Unite! Creating Documentation through Collaboration," Cataloging \& Classification Quarterly 51, no. 1-3 (2013): 214-23. https://doi.org/10.1080/01639374.2012.734367.

7 White, 48-49.

8 Stephanie Schmitt and Sandra Barstow, "Policies and Procedures Manuals in Technical Services: The Forest, the Trees, and the Critters," The Serials Librarian 46, no. 3-4 (April 2004): 257-262, https://doi.org/10.1300/J123v46n03 10.

9 Virginia M. Scheschy, "Cataloging Procedures on the Web: The Greatest Thing Since MARC," Cataloging \& Classification Quarterly, 26:2, 12, https://doi.org/10.1300/J104v26n02 03/.

10 Plummer and Rigda, 25.

11 Ibid., 28.

12 White, 54.

13 Ibid., 54.

14 Ibid., 49.

15 Falk, Hertenstein, and Hunker, 219.

16 Ibid., 220.

${ }^{17}$ Alix Norton, "Where do I Find That?: Creating a Central Shared Documentation System for Publishing Staff Using Google Tools at the University of Michigan Library," Library Hi Tech News 31, 10 (2014): 8-9, https://doi.org/10.1108/LHTN-08-2014-0067.

${ }^{18}$ Plummer and Rigda, 27.

${ }^{19}$ Kristen Blake and Erin Stalberg, "Me and My Shadow: Observation, Documentation, and Analysis of Serials and Electronic Resources Workflow," Serials Review 35, no. 4 (2009): 243, https://doi.org/10.1080/00987913.2009.10765252.

20 Autumn Faulkner, "Lucidchart for Easy Workflow Mapping," Serials Review 44, no. 2 (2018): 157-162, https://doi.org/10.1080/00987913.2018.1472468.

${ }^{21}$ G. Edward Evans, Sheila S. Intner, and Jean Weihs, "Leading the Cataloging Department," in Introduction to Technical Services (Santa Barbara: Libraries Unlimited, 2011), 452-458.
} 
22 Jennifer Turner and Jessica Schomberg, "Inclusivity, Gestalt Principles, and Plain Language in Document Design," In the Library With the Lead Pipe, June 29, 2016, http://www.inthelibrarywiththeleadpipe.org/2016/accessibility/.

23 Luz Rello and Mari-Carmen Marcos, "An Eye Tracking Study on Text Customization for User Performance and Preference," in 2012 Eighth Latin American Web Congress (New York: IEEE, 2012), 64-70, https://doi.org/10.1109/LA-WEB.2012.13.

24 Luz Rello and Ricardo Baeza-Yates, "How to Present More Readable Text for People with Dyslexia," Universal Access in the Information Society 16, no. 1 (2015): 29-49, https://doi.org/10.1007/s10209-0150438-8.

${ }^{25}$ Rello and Marcos, 66.

${ }^{26}$ British Dyslexia Association, "Dyslexia Friendly Style Guide,"accessed June 2, 2019, https://www.bdadyslexia.org.uk/advice/employers/creating-a-dyslexia-friendly-workplace/dyslexia-friendlystyle-guide.

27 Evan Brown, "How to Design Visual Learning Resources for Neurodiverse Students," accessed June 2, 2019, https://blog.fullfabric.com/how-to-design-visual-learning-resources-curriculum-for-neurodiverseaudience-autism-autistic.

${ }^{28}$ W3C Web Accessibility Initiative, "Web Content Accessibility Guidelines (WCAG) Overview," last modified 22 June 2018, https://www.w3.org/WAl/standards-guidelines/wcag/

29 Office of Investor Education and Assistance, U. S. Securities and Exchange Commission, A Plain English Handbook: How to create clear SEC disclosure documents, accessed August 16, 2019, https://www.sec.gov/pdf/handbook.pdf.

${ }^{30}$ Agile Alliance, "Agile 101," accessed June 16, 2019, https://www.agilealliance.org/agile101.

${ }^{31}$ Agile Alliance, "Scrum," accessed June 16, 2019, https://www.agilealliance.org/glossary/scrum.

32 Maria Collins and Kristen Wilson, "An Agile Approach to Technical Services," The Serials Librarian 74 (2018): 9-18, https://doi.org/10.1080/0361526X.2018.1443652.

33 Ibid., 18.

${ }^{34}$ Robin Dean, Elisa Landaverde, Lisa Lorenzo, and Nicole Smeltekop, "Making Library Agile Teams Work" (presentation, Library Information Technology Association Forum, Minneapolis, MN, November 10, 2018), accessed June 28, 2019,

https://docs.google.com/presentation/d/1b51ePfBCOLZaa71bmUsFyxnPd6aU TRudsztj3cR2jA/edit?usp =sharing.

35 Joshua Tauberer, "How to Run a Successful Hackathon," accessed June 28, 2019, https://hackathon.guide/.

36 Joanna DiPasquale, David Keiser-Clark, and Francesca Livermore, "Hack/Doc Partnerships for Library Technology Projects," (presentation, Digital Library Federation Forum, Pittsburgh, PA, October 24, 2017), accessed June 28, 2019, https://drive.google.com/file/d/1OySkajwr8dyW 4-XDMrRjcbablvNYSe2/view.

37 Islandora Collaboration Group, "Templates: How to Run a Hack/Doc," last modified December 5, 2017, https://github.com/Islandora-Collaboration-

Group/icg information/tree/master/templates how to run a hack doc.

${ }^{38}$ Agile Alliance, "12 Principles Behind the Agile Manifesto," accessed August 22, 2019, https://www.agilealliance.org/agile101/12-principles-behind-the-agile-manifesto/.

39 Islandora Collaboration Group.

40 Agile Alliance, "Kanban Board," accessed August 20, 2019, https://www.agilealliance.org/glossary/kanban-board/.

${ }^{41}$ American Library Association, "Knowledge and competencies statements," accessed December 16, 2018, http://www.ala.org/educationcareers/careers/corecomp/corecompspecial/knowledgecompetencies.

42 Association for Library Collections and Technical Services, "Core competencies for cataloging and metadata professional librarians," accessed December 16, 2018,

https://alair.ala.org/bitstream/handle/11213/7853/Core\%20Competencies \%20Cataloging\%20Metadata $\% 2$ OProfessional.pdf?sequence=1\&isAllowed=y.

43 "Why We Care About an Accessible U," Accessible U | Design for All People. All Devices. accessed June 1, 2019, https://accessibility.umn.edu/. 